\title{
Fast DOA Estimation Based on the Transform Domain Weighted Noise Subspace Fitting Algorithm for Generalized Sparse Array
}

\author{
Chunxi Liu $(\mathbb{B}$, Zhikun Chen, and Dongliang Peng $\mathbb{1}$ \\ School of Automation, Hangzhou Dianzi University, Hangzhou 310018, China \\ Correspondence should be addressed to Dongliang Peng; dlpeng@hdu.edu.cn
}

Received 16 March 2021; Revised 4 July 2021; Accepted 15 July 2021; Published 23 July 2021

Academic Editor: Anna Pietrenko-Dabrowska

Copyright ( $) 2021$ Chunxi Liu et al. This is an open access article distributed under the Creative Commons Attribution License, which permits unrestricted use, distribution, and reproduction in any medium, provided the original work is properly cited.

Compared with uniform arrays, a generalized sparse array (GSA) can obtain larger array aperture because of its larger element spacing, which improves the accuracy of DOA estimation. At present, most DOA estimation algorithms are only suitable for the uniform arrays, while a few DOA estimate algorithms that can be applied to the GSA are unsatisfactory in terms of computational speed and accuracy. To compensate this deficiency, an improved DOA estimation algorithm which can be applied to the GSA is proposed in this paper. First, the received signal model of the GSA is established. Then, a fast DOA estimation method is derived by combining the weighted noise subspace algorithm (WNSF) with the concept of "transform domain" (TD). Theoretical analysis and simulation results show that compared with the traditional multiple signal classification (MUSIC) algorithm and the traditional WNSF algorithm, the proposed algorithm has higher accuracy and lower computational complexity.

\section{Introduction}

With the rapid development of information technology, wireless transmission systems, such as mobile communications, radar, and unmanned aerial vehicles (UAV), have become widely used. As such, antenna technology has become more important [1-5], as it has array antenna direction finding, which is an important branch of antenna technology. Most of the existing arrays are uniform arrays, whose element spacing is less than half wavelength of the incident signal, so as to avoid the ambiguity of angle estimation [6-9]. However, when the number of array elements is limited, the aperture of the uniform array will also be affected, which will lead to lower accuracy of DOA estimation $[10,11]$. To solve this problem, scholars have proposed the GSA - a nonequidistant array system - in which the distance between adjacent array elements is more than half of the wavelength of the incident signal [12-15]. Compared with the uniform arrays, the GSA can obtain larger array aperture. Therefore, the estimation accuracy and resolution of the algorithm are effectively improved. The GSA is derived from a uniform array, and it can be obtained by redeploying the array elements of uniform array according to some optimization algorithms (such as simulated annealing algorithm $[16,17]$ and genetic algorithm $[18,19])$. For instance, Chen et al. [20] has proposed an effective method to construct GSA based on the improved genetic algorithm.

The most significant feature of GSA is that the spacing between adjacent array elements is unequal. In this case, most conventional DOA estimation algorithms which have strict requirements on array structure, such as ESPRIT algorithm [21-23], will lose their effect. On the other hand, a few algorithms that are less dependent on the array structure, such as the MUSIC algorithm [24-26], do not perform well in the condition of low SNR. At present, researchers generally use the WNSF algorithm for DOA estimation [27]. The WNSF algorithm has no requirement on array structure and can accurately estimate signal DOA under bad conditions such as low SNR or small number of snapshots [28-30]. However, the WNSF algorithm needs spectral peak search. In accurate search or two-dimensional DOA estimation, the total number of points of the spatial spectrum is very huge, which incurs vast amount of computation and hinders the application of the algorithm in practical engineering [31, 32]. 
To reduce the computation, a reduced-dimension search algorithm was proposed in $[33,34]$, which could reduce the dimensionality of the spectral function and then performed multiple low-dimensional searches. However, this algorithm not only reduced the amount of computation but also reduced the accuracy of parameter estimation. To solve this problem, a concept of transform domain (TD) was proposed $[35,36]$. The authors in [35] transformed the received signal into the coarray domain and then iteratively corrected the phase offset between the coarray data and presumed model caused by angle biases according to a closed-form formula. On the other hand, Zhang et al. [36] used spherical Fourier domain to construct the array signal model. However, these methods are only applicable to uniform liner arrays, which means that the method is not universal. Nevertheless, it is a concept worth learning and developing.

Motivated by the above facts, in this paper, we combine the concept of "transform domain" with the WNSF algorithm to obtain a fast DOA estimation method which is suitable for the GSA. The procedure of the proposed method is as follows. Firstly, we establish the received data model of the GSA. Secondly, the model is transformed into the TD. Then, the intersection of the noise subspace and its conjugate space in the TD is used to replace the noise subspace in the spectral search function. In this manner, the calculation range of the search function can be reduced by half, and the "rapidity" of the algorithm can be achieved. The theoretical analysis and computer simulation results show that the proposed algorithm has higher accuracy and lower computational complexity than the MUSIC algorithm and traditional WNSF algorithm.

\section{Signal Model of GSA}

Consider a GSA composed of $M$ elements, as shown in Figure 1. The array elements are arbitrarily distributed in space, and the position coordinates of the array elements are given by $\left(x_{m}, y_{m}, z_{m}\right), \quad m=1,2, \ldots, M$.

The black dots in Figure 1 represent the array elements. The elevation angle $\theta$ is defined as the intersection angle between the signal and the positive direction of $Z$-axis, while the azimuth angle $\varphi$ is defined as the angle between the projection of the signal onto the $X O Y$ plane and the positive direction of the $X$-axis. Here, the value ranges of $\theta$ and $\varphi$ are both $(-\pi / 2, \pi / 2)$. Assume that $K$ narrow-band waves impinge upon the GSA from the elevation angle $\theta_{k}$ and the azimuth angle $\varphi_{k}$, the data received by a snapshot of the array can be expressed as

$$
x(t)=A(\theta, \varphi) s(t)+n(t),
$$

where $x(t) \triangleq\left[x_{1}(t), x_{2}(t), \cdots, x_{M}(t)\right]^{T}$ is the $M \times 1$ dimensional array receiving data; $s(t) \triangleq\left[s_{1}(t), s_{2}(t), \cdots\right.$, $\left.s_{K}(t)\right]^{T}$ is the signal sampling data; and $n(t)$ is the additive white Gaussian noise matrix with the same dimension as $x(t)$. The array steering matrix $A(\theta, \varphi) \triangleq\left[a\left(\theta_{1}, \varphi_{1}\right)\right.$, $\left.a\left(\theta_{2}, \varphi_{2}\right), \ldots, a\left(\theta_{K}, \varphi_{K}\right)\right]$ and the steering vector $a\left(\theta_{k}, \varphi_{k}\right)$ can be expressed as

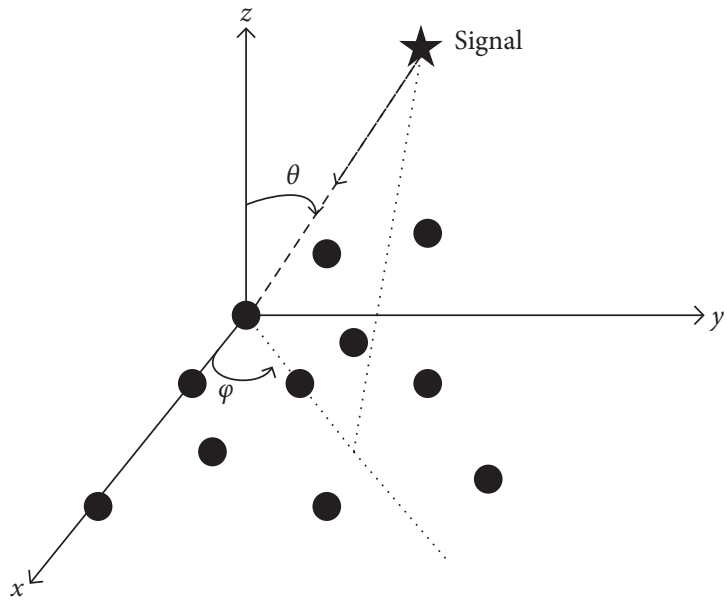

FIgURE 1: Generalized sparse array.

$$
a\left(\theta_{k}, \varphi_{k}\right) \triangleq\left[e^{j \beta_{k, 1}}, e^{j \beta_{k, 2}}, \ldots, e^{j \beta_{k, M}}\right]
$$

where $j=\sqrt{-1}$ is the plural unit. Define $\lambda$ as the signal wavelength. Then, $\beta_{k, m}$ is given by

$$
\beta_{k, m}=\frac{2 \pi}{\lambda}\left(x_{m} \sin \theta_{k} \cos \varphi_{k}+y_{m} \sin \theta_{k} \sin \varphi_{k}+z_{m} \cos \theta_{k}\right) \text {. }
$$

The process of estimating the $2 \mathrm{D}$ spatial angle of the $K$ signals based on the signal model constructed by (1) is called spatial spectral estimation.

\section{DOA Estimation Algorithm Based on TD- WNSF}

3.1. Principle of the Traditional WNSF Algorithm. In practical application, according to the received data from $P$ snapshots, the spatial correlation is estimated using a time average, and the covariance matrix of the array output is obtained by

$$
\widehat{R}_{\mathrm{XX}}=\frac{1}{P} \mathrm{XX}^{H},
$$

where $X$ is the received data matrix of $P$ snapshots. After performing an eigenvalue decomposition, we can derive the following result:

$$
\widehat{R}_{X X}=U_{S} D_{S} U_{S}^{H}+U_{N} D_{N} U_{N}^{H},
$$

where $U_{S}$ is the signal subspace composed of eigenvectors corresponding to $K$ larger eigenvalues and $U_{N}$ is the noise subspace composed of eigenvectors corresponding to $M-K$ smaller eigenvalues. Accordingly, we can deduce that the signal subspace and the space formed by the steering vector of the array are the same, and the steering vector space of the array and the noise subspace are orthogonal to each other [37]. This orthogonal relation can be expressed as

$$
a^{H}(\theta, \varphi) U_{N}=O_{M \times 1},
$$


where the symbol ' $H$ ' represents the conjugate transpose operation, $a(\theta, \varphi)$ is the $M \times 1$ steering vector, and $O$ is the $1 \times M-K$ zero vector.

Considering that the length of the actual received data matrix is limited and noise is mixed in the actual received data matrix, $a(\theta, \varphi)$ and $U_{N}$ are not completely orthogonal. In other words, equation (6) is not completely valid. Therefore, the following formula of noise subspace fitting (NSF) is considered [38]:

$$
\begin{aligned}
(\theta, \varphi) & =\min \left\|U_{N}^{H} A(\theta, \varphi)\right\|_{F}^{2} \\
& =\min \operatorname{tr}\left\{U_{N}^{H} A(\theta, \varphi) A^{H}(\theta, \varphi) U_{N}\right\} .
\end{aligned}
$$

Equation (7) can be further extended to a weighted form. The relationship between the noise subspace and the steering vector space of the array is given by

$$
U_{N}^{H} A(\theta, \varphi) W^{(1 / 2)}=0
$$

where $W$ is the weight. Then, the fitting formula given by equation (7) can be transformed into

$$
(\theta, \varphi)=\min \left\|U_{N}^{H} A(\theta, \varphi) W^{1 / 2}\right\|_{F}^{2}=\min \operatorname{tr}\left\{U_{N}^{H} A(\theta, \varphi) W A^{H}(\theta, \varphi) U_{N}\right\}=\min \operatorname{tr}\left\{W A^{H}(\theta, \varphi) U_{N} U_{N}^{H} A(\theta, \varphi)\right\} .
$$

Theoretically, the DOA estimation in equation (9) can be estimated by bringing a $2 \mathrm{D}$ search to bear on the ranges of all parameters; however, this is computationally exhaustive.

3.2. TD-WNSF Algorithm. When running the traditional WNSF algorithm, an extreme value test should be conducted for each point in the spatial spectrum. With the improvement in search accuracy, the number of spectral points is increased further, leading to a sharp increase in the running time of the algorithm. If we can find a way to compress the extremum search range, then the speed of DOA estimation can also be improved. From this analysis, the following transformation is considered:

$$
\left\{\begin{array}{l}
u=\sin \theta \cos \varphi \\
v=\sin \theta \sin \varphi \\
\sqrt{1-u^{2}-v^{2}}=\cos \theta .
\end{array}\right.
$$

Combining equations (3) and (10) leads to

$$
\beta_{k, m}=\frac{2 \pi}{\lambda}\left(x_{m} u_{k}+y_{m} v_{k}+z_{m} \sqrt{1-u_{k}^{2}-v_{k}^{2}}\right) \text {. }
$$

Moreover, combining equations (2) and (11) leads to

$$
a(u, v)=\left[\begin{array}{c}
e^{j 2 \pi\left(x_{1} u+y_{1} v+z_{1} \sqrt{1-u^{2}-v^{2}}\right)} \\
e^{j 2 \pi\left(x_{2} u+y_{2} v+z_{2} \sqrt{1-u^{2}-v^{2}}\right)} \\
\vdots \\
e^{j 2 \pi\left(x_{M} u+y_{M} v+z_{M} \sqrt{1-u^{2}-v^{2}}\right)}
\end{array}\right]=\left[\begin{array}{c}
e^{j 2 \pi\left(x_{1}(-u)+y_{1}(-v)+z_{1} \sqrt{1-(-u)^{2}-(-v)^{2}}\right)} \\
e^{j 2 \pi\left(x_{2}(-u)+y_{2}(-v)+z_{2} \sqrt{1-(-u)^{2}-(-v)^{2}}\right)} \\
\vdots \\
e^{j 2 \pi\left(x_{M}(-u)+y_{M}(-v)+z_{M} \sqrt{1-(-u)^{2}-(-v)^{2}}\right)}
\end{array}\right]^{*}(-u,-v),
$$

where ${ }^{*}$ represents the conjugate operation. The steering vector $a(\theta, \phi)$ and the transformation domain steering vector $a(u, v)$ are clearly equivalent in a physical sense. Then, $(-u,-v)$ in equation (12) can be depicted as a virtual mirror signal source symmetry to $(u, v)$. Assume that the weight is given by $W=I$, where $I$ represents the identity matrix. Moreover, combining equations (6) and (12) leads to

$$
\left\{\begin{array}{l}
a^{H}(u, v) U_{N}=O, \\
{\left[a^{*}(u, v)\right]^{H} U_{N}^{*}=a^{H}(-u,-v) U_{N}^{*}=O .}
\end{array}\right.
$$

In equation (13), the steering vector corresponding to the real signal source $(u, v)$ is orthogonal to the noise subspace $U_{N}$, whereas the steering vector corresponding to the virtual mirror signal source $(-u,-v)$ is orthogonal to the conjugate noise subspace $U_{N}^{*}$. If we replace the noise space in the WNSF algorithm with the intersection space of $U_{N}$ and $U_{N}^{*}$, as the intersection space is orthogonal to the real steering vector and the virtual steering vector, then the WNSF algorithm can generate an extremum at the real signal source and virtual signal source simultaneously. This characteristic means that the DOA estimation only needs to search half of the $(u, v)$ domain. Therefore, the purpose of "rapidity" can be achieved. This algorithm of constructing the WNSF spectral function in the TD is called the TD-WNSF algorithm.

Solving the intersection space of $U_{N}$ and $U_{N}^{*}$ is essential to constructing the TD-WNSF algorithm. The steps discussed briefly describe the method to find the intersection space of the two subspaces.

First, we define a concept called "adjoint solution." Suppose that $\left[\alpha_{1}, \alpha_{2}, \ldots, \alpha_{s}\right]$ and $\left[\beta_{1}, \beta_{2}, \ldots, \beta_{t}\right]$ are two groups of vectors of the linear space $V$ and $\left(a_{1}, a_{2}, \ldots, a_{s}, b_{1}, b_{2}, \ldots, b_{t}\right)$ is a solution of the equation 
$x_{1} \alpha_{1}+x_{2} \alpha_{2}+\ldots+x_{s} \alpha_{s}=y_{1} \beta_{1}+y_{2} \beta_{2}+\ldots+y_{t} \beta_{t}$. Then, $\left(a_{1}, a_{2}, \cdots, a_{s}\right)$ is called the adjoint solution of $\left(a_{1}, a_{2}, \ldots, a_{s}, b_{1}, b_{2}, \ldots, b_{t}\right)$.

Suppose that the noise subspace $U_{N}$ is represented by $\left[\alpha_{1}, \alpha_{2}, \ldots, \alpha_{M-K}\right]$ and the conjugate noise subspace $U_{N}^{*}$ is represented by $\left[\beta_{1}, \beta_{2}, \ldots, \beta_{M-K}\right]$. Then, the intersection space $U_{\text {inter }}$ of $U_{N}$ and $U_{N}^{*}$ can be determined by the adjoint solution of

$$
x_{1} \alpha_{1}+x_{2} \alpha_{2}+\ldots+x_{s} \alpha_{s}=y_{1} \beta_{1}+y_{2} \beta_{2}+\ldots+y_{t} \beta_{t},
$$

which is specifically expressed by Definition 1 in this study.

Definition 1. $U_{\text {inter }}=U_{N} \cap U_{N}^{*}=\left\{k_{1} \alpha_{1}+k_{2} \alpha_{2}+\ldots+k_{s} \alpha_{s} \mid\right.$ $\left.\left(k_{1}, k_{2}, \ldots, k_{s}\right)\right\}$ is the adjoint solution of the solution of equation (14).

The definition can be proven as follows. Suppose $\zeta=U_{\text {inter. }}$ From $\zeta \in U_{N}$, we can obtain

$$
\zeta=k_{1} \alpha_{1}+k_{2} \alpha_{2}+\ldots+k_{s} \alpha_{s}
$$

and from $\zeta \in U_{N}^{*}$, we can derive

$$
\zeta=l_{1} \beta_{1}+l_{2} \beta_{2}+\ldots+l_{s} \beta_{s} .
$$

Thus, we have

$$
k_{1} \alpha_{1}+k_{2} \alpha_{2}+\ldots+k_{s} \alpha_{s}=l_{1} \beta_{1}+l_{2} \beta_{2}+\ldots+l_{s} \beta_{s} .
$$

Consequently, $\left(k_{1}, k_{2}, \ldots, k_{s}\right)$ is an adjoint solution of equation (14).

By contrast, if $\left(k_{1}, k_{2}, \ldots, k_{s}\right)$ is the adjoint solution of a solution to that of equation (14), then $\left(k_{1}, k_{2}, \ldots\right.$, $\left.k_{s}, l_{1}, l_{2}, \ldots, l_{s}\right)$ is the solution of equation (14), which is also the expression of equation (17). Given that the left side of equation (17) belongs to $U_{N}$, whereas the right side of equation (17) belongs to $U_{N}^{*}$, we have

$$
\zeta=k_{1} \alpha_{1}+k_{2} \alpha_{2}+\ldots+k_{s} \alpha_{s} \in U_{\text {inter }} .
$$

In summary, $U_{\text {inter }}=U_{N} \cap U_{N}^{*}=\left\{k_{1} \alpha_{1}+k_{2} \alpha_{2}+\cdots+\right.$ $\left.k_{s} \alpha_{s} \mid\left(k_{1}, k_{2}, \cdots, k_{s}\right)\right\}$ is the adjoint solution of the solution of equation (14). At this stage, the proof has been completed.

On the basis of on the above algorithm, the TD-WNSF spectrum can be defined as

$$
f_{\mathrm{TD}-\mathrm{WNSF}}(u, v)=\frac{1}{\operatorname{tr}\left\{W A^{H}(u, v) U_{\text {inter }} U_{\text {inter }}^{H} A(u, v)\right\}} .
$$

As mentioned, this TD-WNSF spectral function generates extreme values at the real and mirror positions of the signal at the same time. Thus, only a half-domain search in the $(u, v)$ domain is needed to estimate the signal direction. Then, an accurate search is performed at the vicinity of the spectral peak position and its mirror image position to obtain the precise arrival angle. On the basis of the previous analysis, the proposed algorithm does not entail any array structure requirement and can be applied to the GSA.
3.3. Description of Algorithm Step. The implementation steps of the proposed method are summarized as follows:

(i) Step 1: perform eigenvalue decomposition of the array-received data in order to obtain the noise subspace.

(ii) Step 2: calculate the intersection space based on the algorithm of Definition 1 and then construct the TD-WNSF spectrum based on equation (19).

(iii) Step 3: search the positive half-spectrum of equation (18) to obtain the estimated value $\left(\widehat{u}_{i}, \widehat{v}_{i}\right)$ of the DOA parameter in the $(u, v)$ domain, where $i=1,2, \ldots, K$.

(iv) Step 4: replace $a(\theta, \varphi)$ in equation (6) with $a\left(\widehat{u}_{i}, \widehat{v}_{i}\right)$ and for the extreme value test. Among the two elements, the one that satisfies $a^{H}(u, v) U_{N}=0$ is the real TD-DOA.

(v) Step 5: substitute the TD-DOA, which was obtained in step 4 , into equation (10) to calculate the spatial $\operatorname{DOA}\left(\widehat{\theta}_{i}, \widehat{\varphi}_{i}\right)$.

(vi) Step 6: perform an accurate searching in a small area near $\left(\widehat{\theta}_{i}, \widehat{\varphi}_{i}\right)$. The element that satisfies $a^{H}(\theta, \varphi) U_{N}=0$ is the real spatial domain DOA.

As shown in these steps, the algorithm first implements a rough search process to obtain the TD-DOA $\left(\widehat{u}_{i}, \widehat{v}_{i}\right)$. Then, the inverse trigonometric function is transformed to calculate the rough estimation of the angle $\left(\widehat{\theta}_{i}, \widehat{\varphi}_{i}\right)$. Finally, an accurate search is conducted in the small neighborhood of $\left(\widehat{\theta}_{i}, \widehat{\varphi}_{i}\right)$ to obtain the fine estimate $\left(\widehat{\theta}_{i}, \widehat{\varphi}_{i}\right)$. As such, no angle measurement blurring will occur.

3.4. Algorithm Complexity Analysis. The traditional MUSIC algorithm, the traditional WNSF algorithm, and the TDWNSF algorithm are compared in this study. The array structure used by the three algorithms is shown in Figure 1. Consider that $K$ uncorrelated signals impinge upon an GSA of $M$ elements, the number of snapshots is given by $L$, and the number of search points is given by $q$. Assume that all the three algorithms perform a rough search, which means that the step size is $1^{\circ}$.

For the traditional MUSIC algorithm, the modulus $\left\|a^{H}(\theta, \varphi) U_{N}\right\|^{2}$ needs to be calculated for each spectral point, and the dimension of $U_{N}$ is $M \times(M-K)$. Therefore, the computation of the spectral search of the traditional MUSIC algorithm is $q(M-K)(M+1)$. The computation of the eigenvalue decomposition in the $M \times M$ dimensional autocovariance matrix is $M(K+2)^{2}$. Therefore, the total computation of the traditional MUSIC algorithm is $q(M-K)(M+1)+M(K+2)^{2}$.

For the traditional WNSF algorithm, the weight $W$ is usually taken as a unit matrix $I$ in the calculation. The operation used to find the $\operatorname{trace} \operatorname{tr}\left(\left\|a^{H}(\theta, \varphi) U_{N}\right\|^{2}\right)$ is needed to calculate each spectral point. The dimension of $U_{N}$ is $M \times(M-K)$, and the computation involved in finding the trace is $q(M-K)(M+M)$. The computation of the matrix's 
eigenvalue decomposition is the same as that of the traditional MUSIC algorithm, which is $M(K+2)^{2}$. Therefore, the total computation of the traditional MUSIC algorithm is $q(M-K)(M+M)+M(K+2)^{2}$.

For the TD-WNSF algorithm proposed in this study, the operation used to find the $\operatorname{trace} \operatorname{tr}\left(\left\|a^{H}(\theta, \varphi) U_{\text {inter }}\right\|^{2}\right)$ is needed to calculate each spectral value point. The intersection space $U_{\text {inter }}$ has a lower dimension denoted by $M \times$ $(M-2 K)$ compared with $U_{N}$, and the search range of the TD-WNSF algorithm is reduced by half. Thus, the computation of the peak search is $q(M-2 K)(M+1) / 2$, while the computation of the eigenvalue decomposition for the autocovariance matrix in the TD-MUSIC is $2(M-1) \times(K-1)^{2}$. Therefore, the total computation of the TD-MUSIC algorithm is $q(M-2 K)(M+1) /$ $2+2(M-1)(K-1)^{2}$.

Table 1 shows a comparison of the computations of the three algorithms with the number of array elements.

We can easily see that, with the increase of the number of array elements, the computational complexity of our algorithm is far lower than the other two algorithms, which reflects the rapidity of our algorithm.

\section{Simulation and Analysis}

Consider a generalized sparse array with 16 array elements, the position distribution of its array elements is shown in Table 2.

4.1. Simulation 1: Signal DOA Parameter Estimation. This simulation was implemented to evaluate the effectiveness of the proposed TD-WNSF algorithm. Considering a signal with the following values, $\theta=60^{\circ}$ and $\varphi=20^{\circ}$, impinging upon the GSA. SNR is taken to be $0 \mathrm{~dB}$. The running result of our algorithm is shown in Figure 2. From Figure 2, we can see that the estimation of TD-DOA is $(0.17,0.865)$. According to equation (10), the spatial angle $\left(60^{\circ}, 20^{\circ}\right)$ and the TD angle $(0.17,0.865)$ are equivalent, which illustrates that our algorithm can accurately measure the DOA of the signal.

4.2. Simulation 2: Relationship between Algorithm Performance and SNR. This simulation is implemented to compare the DOA estimation performances of the MUSIC, WNSF, and TD-WNSF algorithms. The Monte Carlo number is $L=500$, and the SNR shifts from -10 to $20 \mathrm{~dB}$. The rootmean-squared error (RMSE) is defined as

$$
\left\{\begin{array}{l}
\operatorname{RMSE}_{\theta}=\frac{1}{K} \sum_{k=1}^{K} \sqrt{\frac{1}{L} \sum_{l=1}^{L}\left(\widehat{\theta}_{k, l}-\theta_{k}\right)^{2}}, \\
\operatorname{RMSE}_{\varphi}=\frac{1}{K} \sum_{k=1}^{K} \sqrt{\frac{1}{L} \sum_{l=1}^{L}\left(\hat{\varphi}_{k, l}-\varphi_{k}\right)^{2}},
\end{array}\right.
$$

TABle 1: Computations of the three algorithms.

\begin{tabular}{lcccccc}
\hline Array number & 5 & 6 & 7 & 8 & 9 & 10 \\
\hline MUSIC & 3320 & 5136 & 6952 & 9848 & 12744 & 16000 \\
WNSF & 5480 & 8640 & 12712 & 17408 & 22824 & 28960 \\
TD-WNSF & 548 & 1270 & 2172 & 3254 & 4516 & 5958 \\
\hline
\end{tabular}

TABLE 2: The position coordinates of the array.

\begin{tabular}{lccc}
\hline \multicolumn{3}{c}{ Coordinate } \\
Number & $\mathrm{X}(m)$ & $\mathrm{Y}(m)$ & $\mathrm{Z}(m)$ \\
\hline 1 & 0 & 0 & 0 \\
2 & -0.04487 & 0.12177 & 0.12131 \\
3 & -0.08974 & -0.24355 & 0.24264 \\
4 & -0.13461 & -0.36533 & 0.36395 \\
5 & 0.07681 & -0.04924 & 0.10906 \\
6 & 0.03194 & -0.17101 & 0.23038 \\
7 & -0.01293 & -0.29279 & 0.35170 \\
8 & -0.05780 & -0.41456 & 0.47302 \\
9 & 0.19849 & 0.23309 & 0.09681 \\
10 & 0.15362 & -0.98460 & 0.21812 \\
11 & 0.10875 & -0.22024 & 0.33945 \\
12 & 0.63880 & -0.34202 & 0.46076 \\
13 & 0.27529 & -0.25930 & 0.20587 \\
14 & 0.23043 & -0.14769 & 0.32719 \\
15 & 0.18556 & -0.26948 & 0.44851 \\
16 & 0.14069 & -0.39125 & -0.39125 \\
\hline
\end{tabular}

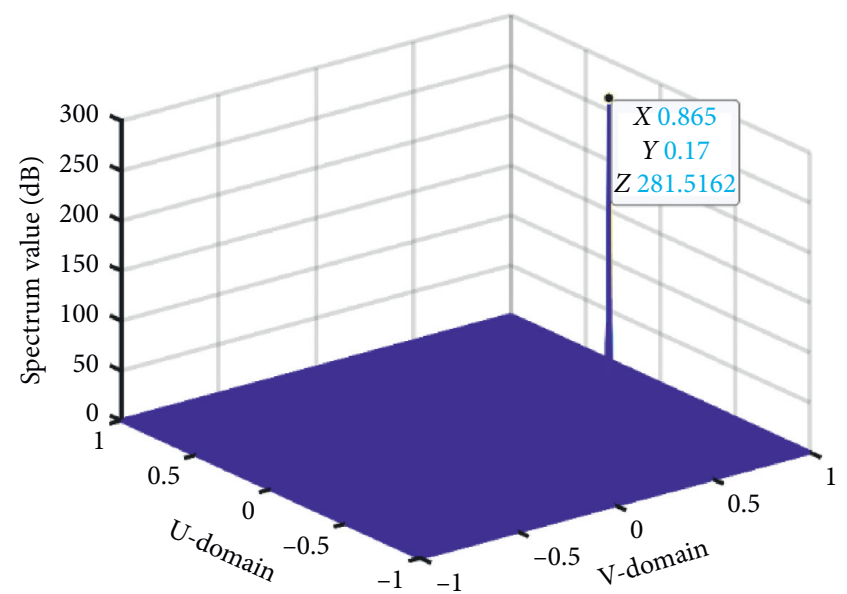

Figure 2: TD-WNSF spectrum.

where $\widehat{\theta}_{k, l}$ and $\widehat{\varphi}_{k, l}$ represent the estimated values of the $K$ th signal in the Lth Monte Carlo simulation, respectively. Figure 3 shows the relationship between the RMSE of the azimuth angle and SNR, while Figure 4 shows the relationship between the RMSE of the elevation angle and SNR.

From Figures 3 and 4, we can see that the RMSE curves of three methods decrease as SNR increases. However, the RMSE curve of our TD-WNSF algorithm locates below that of MUSIC and WNSF all along, which indicates that the TDWNSF is superior to MUSIC and RD-MUSIC in a different SNR. This is because our method performs many finegrained searches in the neighborhood of the TD estimation. 


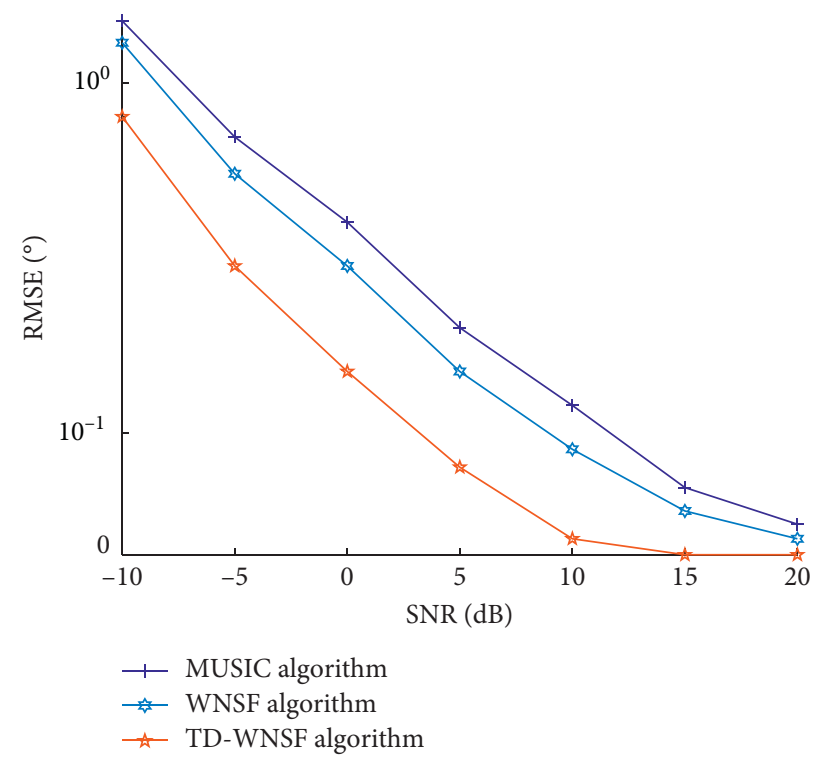

FIgURE 3: RMSE of the azimuth angle.

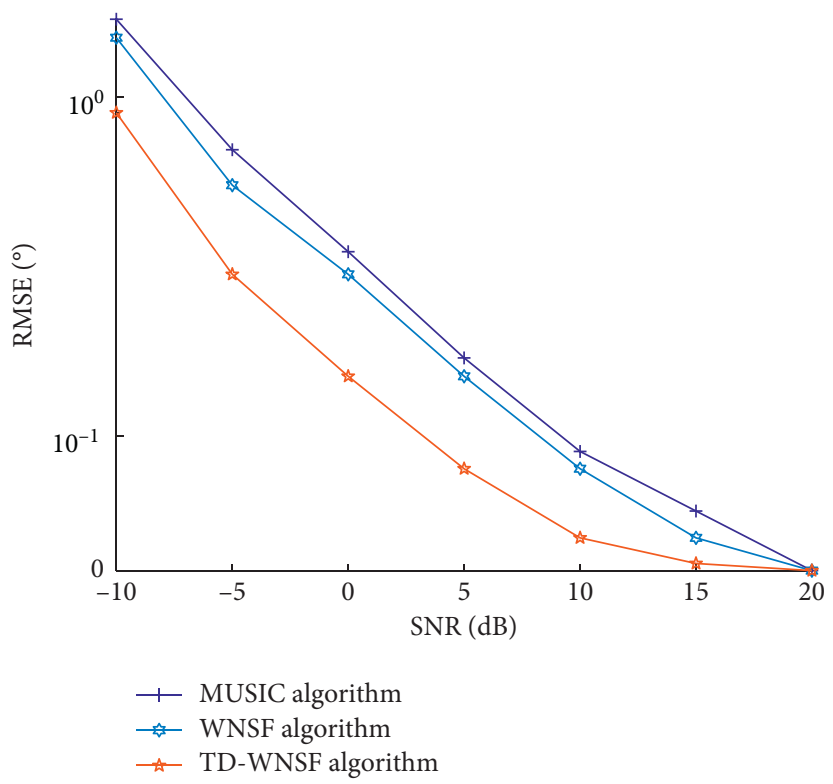

FIgURE 4: RMSE of the elevation angle.

4.3. Simulation 3: Relationship between Algorithm Performance and the Angle of Incident Signal. To observe the performance of the new approach more clearly, we compared the algorithm performance with the angle of incident signal. The simulation consists of two parts. The first part entails fixing the elevation angles to $15^{\circ}, 30^{\circ}, 45^{\circ}, 60^{\circ}$, and $75^{\circ}$. The change in azimuth angle is conducted to explore the relationship between RMSE and azimuth angle. The second part involves fixing the azimuth angles to $15^{\circ}, 30^{\circ}, 45^{\circ}, 60^{\circ}$, and $75^{\circ}$. The change in elevation angle is performed to explore the relationship between RMSE and elevation angle.

The simulation result of the first part is shown in Figure 5.
Figure 5 shows the relationship between the performance of algorithms and the azimuth angle of the incident signal under five typical elevation angles. The comparison of the five subgraphs indicates that the array has high sensitivity to the signals with azimuth angle between $0^{\circ}$ and $40^{\circ}$.

The simulation result of the second part is shown in Figure 6.

Figure 6 shows the relationship between the performance of algorithms and the incident elevation angle of the incident signal under five typical azimuth angles. The comparison of the five subgraphs indicates that the array has high sensitivity to the signals with an elevation angle between $10^{\circ}$ and $35^{\circ}$. 


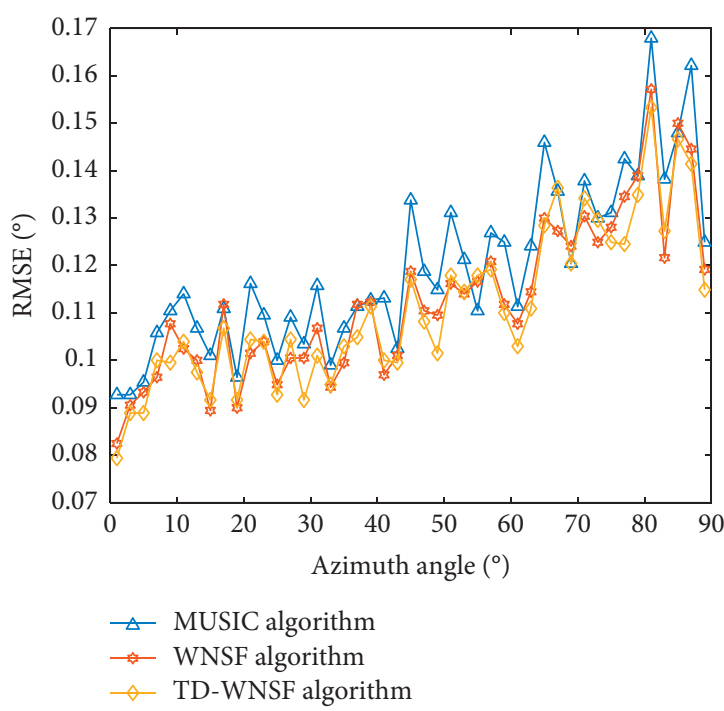

(a)

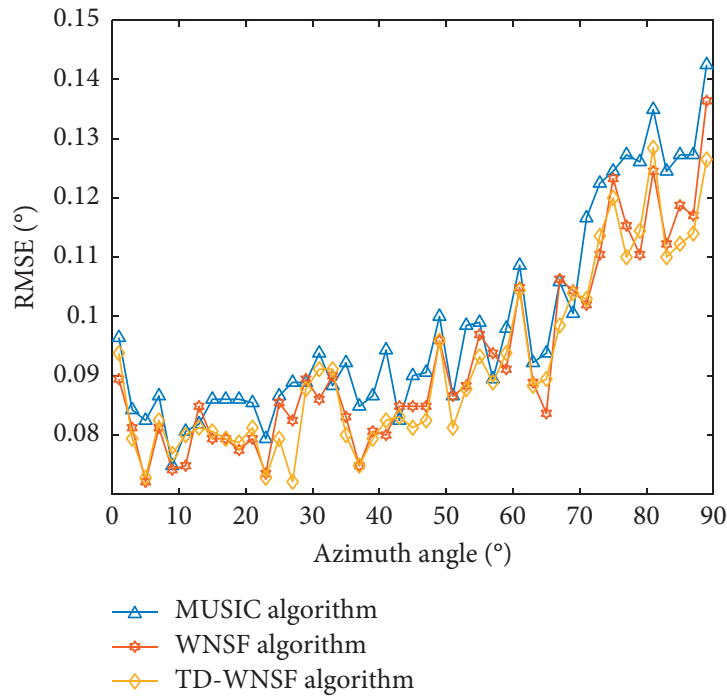

(c)

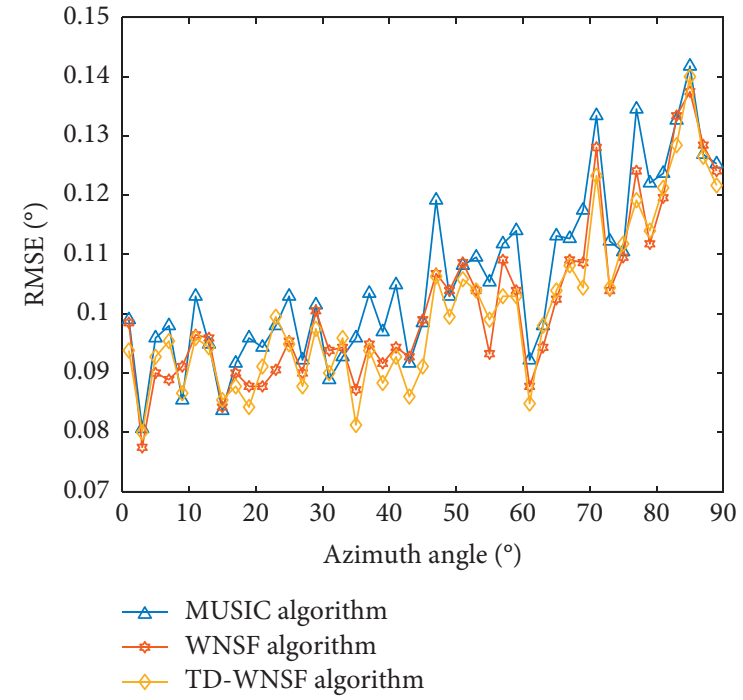

(b)

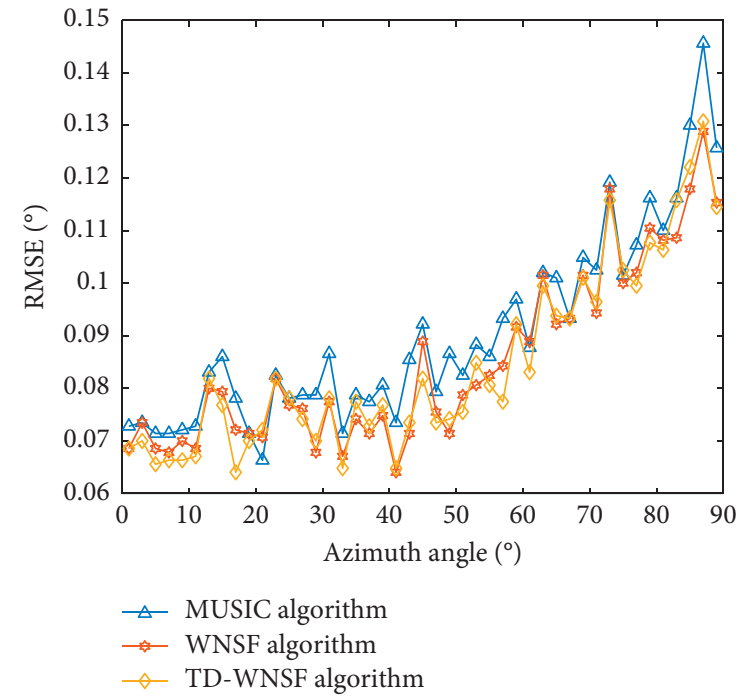

(d)

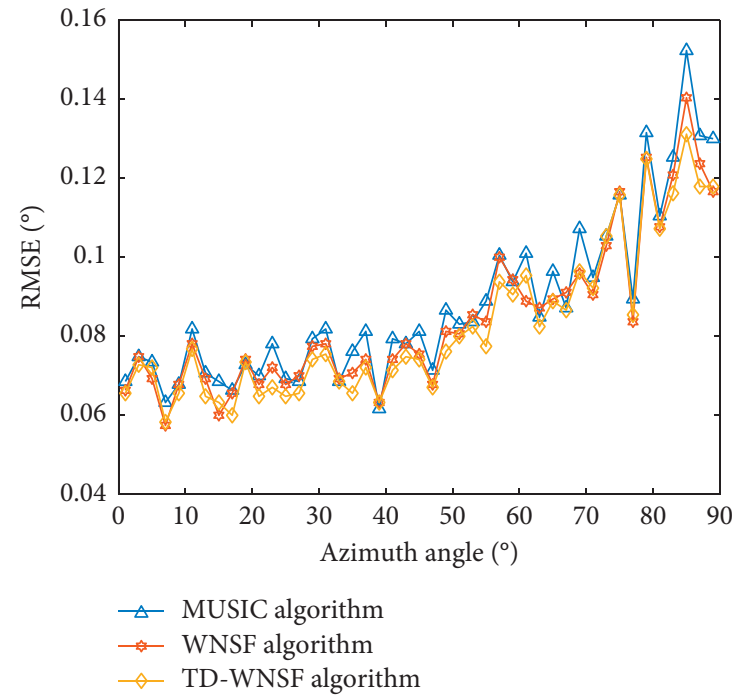

(e)

Figure 5: Relationship between RMSE and azimuth angle at different elevation angles. (a) Fixed elevation angle of $15^{\circ}$. (b) Fixed elevation angle of $30^{\circ}$. (c) Fixed elevation angle of $45^{\circ}$. (d) Fixed elevation angle of $60^{\circ}$. (e) Fixed elevation angle of $75^{\circ}$. 

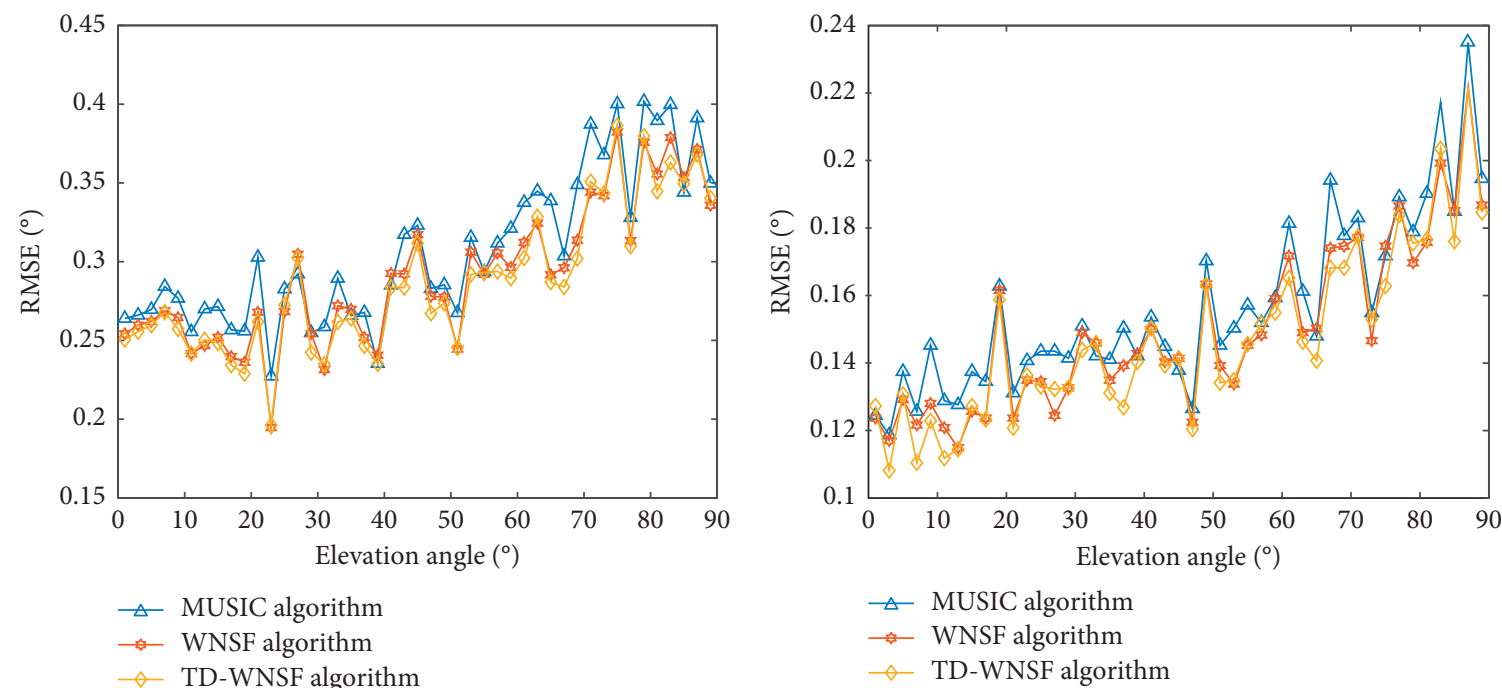

(a)

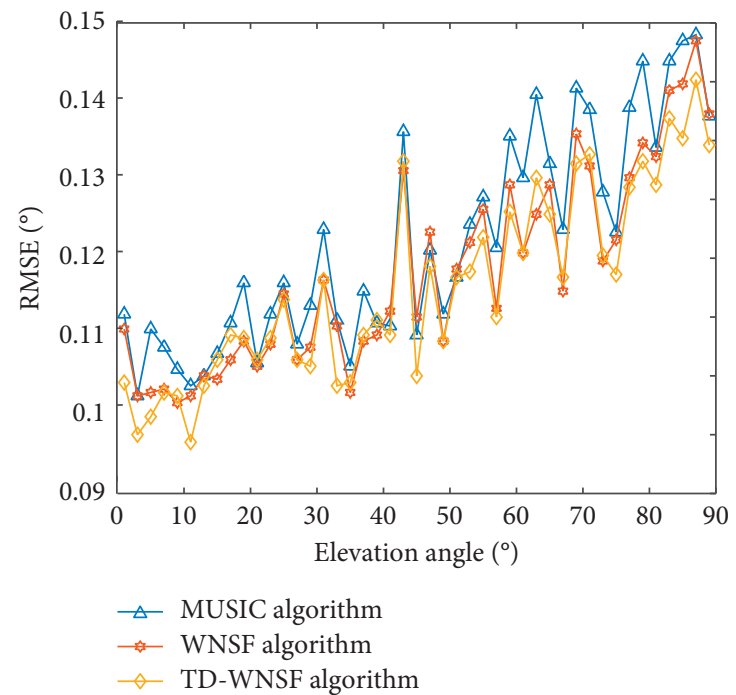

(c)

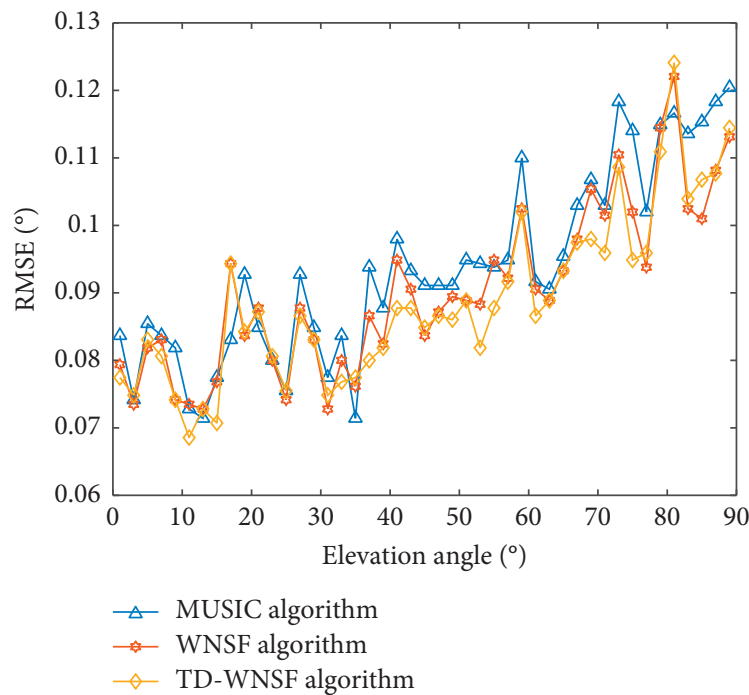

(d)

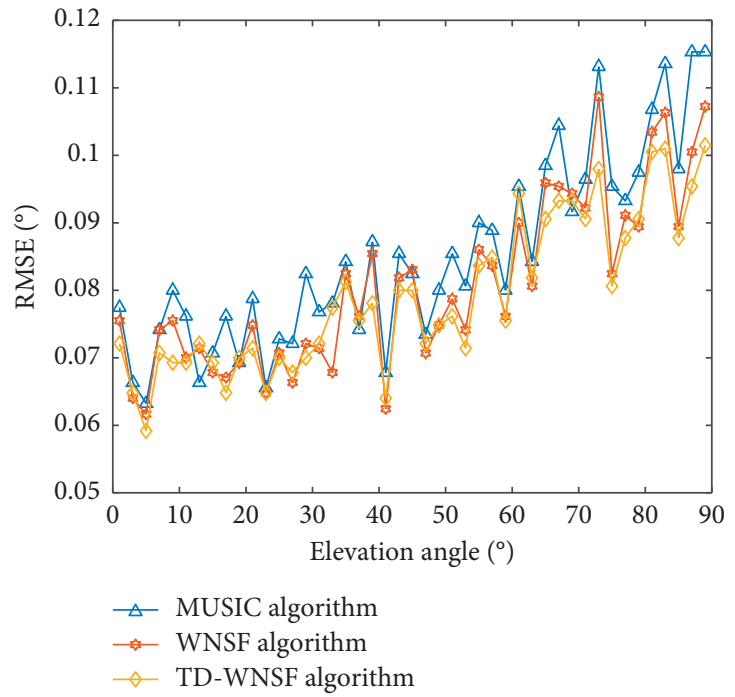

(e)

Figure 6: Relationship between RMSE and elevation angle at different azimuth angles. (a) Fixed azimuth angle of $15^{\circ}$. (b) Fixed azimuth angle of $30^{\circ}$. (c) Fixed azimuth angle of $45^{\circ}$. (d) Fixed azimuth angle of $60^{\circ}$. (e) Fixed azimuth angle of $75^{\circ}$. 


\section{Conclusions}

In this paper, a novel and efficient TD-WNSF algorithm for estimating the DOA was proposed, which can be applied to the GSA. We first established the received data model of the GSA. And then, we exploited the symmetry of the fitting estimator in the TD to reduce the spectral searching range. Compared with the traditional MUSIC and WNSF algorithms, the computational complexity of the proposed algorithm is significantly reduced. The simulation results indicate that the TD-WNSF algorithm has high accuracy and efficiency.

\section{Data Availability}

The data that support the findings of this study are available from the corresponding author upon reasonable request.

\section{Conflicts of Interest}

The authors declare that there are no conflicts of interest regarding the publication of this paper.

\section{Acknowledgments}

This work was supported by the Natural Science Foundation of China (grant no. 61701148).

\section{References}

[1] X. Lin, X. Zhang, L. He, and W. Zheng, "Multiple emitters localization by UAV with nested linear array: system scheme and 2D-DOA estimation algorithm," China Communications, vol. 17, no. 3, pp. 117-130, 2020.

[2] H. Krim and M. Viberg, "Two decades of array signal processing research: the parametric approach," IEEE Signal Processing Magazine, vol. 13, no. 4, pp. 67-94, 1996.

[3] H. L. Van Trees, "Detection, estimation, and modulation theory," Optimum Array Processing, pp. 55-89, WileyInterscience, New York, NY, USA, 2004.

[4] Z. Zhou, Z. Wei, Z. Tang, and Y. Yin, "Design and analysis of a wideband multiple-microstrip dipole antenna with high isolation," IEEE Antennas and Wireless Propagation Letters, vol. 18, no. 4, pp. 722-726, 2019.

[5] Y.-H. Yang, B.-H. Sun, and J.-L. Guo, "A single-layer wideband circularly polarized antenna for millimeter-wave applications," IEEE Transactions on Antennas and Propagation, vol. 68, no. 6, pp. 4925-4929, 2020.

[6] J. Liu, "Evaluation on the MUSIC algorithm performance of uniform rectangular arrays," in Proceedings of the 2019 International Conference on Microwave and Millimeter Wave Technology (ICMMT), pp. 1-3, Guangzhou, China, May 2019.

[7] C. Sun and J. Ding, "Application of the DOA algorithms in the uniform circular array antennas," in Proceedings of the 2018 International Conference on Intelligent Transportation, Big Data \& Smart City (ICITBS), pp. 633-636, Xiamen, China, January 2018.

[8] Z.-S. Qi, Y. Guo, B.-H. Wang, and C. Gong, "The performance analysis of MUSIC algorithm based on Conformal array antenna," Journal of Electronics \&information Technology, vol. 30, no. 11, pp. 2674-2677, 2008.

[9] W. Shan, J. Wang, X. kai Jia, J. Wang, and J. Guan, "Research on uniform linear array output signal and beamforming," in
Proceedings of the 2020 IEEE International Conference on Signal Processing, Communications and Computing (ICSPCC), pp. 1-4, Xi'an, China, August 2020.

[10] F. Keskin and T. Filik, "An optimum volumetric array design approach for both azimuth and elevation isotropic DOA estimation," IEEE Access, vol. 8, pp. 183903-183912, 2020.

[11] S. Mazlout, M.-B. B. Salah, and A. Samet, "Decision-based algorithm for robust 2D-DoA estimation with L-shaped array," in Proceedings of the 2019 15th International Wireless Communications \& Mobile Computing Conference (IWCMC), pp. 391-396, Tangier, Morocco, June 2019.

[12] Y. Gong, S. Xiao, and B.-Z. Wang, "Synthesis of sparse planar arrays with multiple patterns by the generalized matrix enhancement and matrix pencil," IEEE Transactions on Antennas and Propagation, vol. 69, no. 2, pp. 869-881, 2021.

[13] G. Oliveri, M. Carlin, and A. Massa, "Complex-weight sparse linear array synthesis by bayesian compressive sampling," IEEE Transactions on Antennas and Propagation, vol. 60, no. 5, pp. 2309-2326, 2012.

[14] F. Viani, G. Oliveri, and A. Massa, "Compressive sensing pattern matching techniques for synthesizing planar sparse arrays," IEEE Transactions on Antennas and Propagation, vol. 61, no. 9, pp. 4577-4587, 2013.

[15] A. A. Khan and A. K. Brown, "Null synthesis in irregularly spaced sparse antenna arrays," in Proceedings of the 2013 IEEE International Symposium on Phased Array Systems and Technology, pp. 564-566, Boston, MA, USA, October 2013.

[16] X. Zhihong, S. Bo, and G. Yanyan, "Using simulated annealing and ant colony hybrid algorithm to solve traveling salesman problem," in Proceedings of the 2009 Second International Conference on Intelligent Networks and Intelligent Systems, pp. 507-510, Tianjin, China, November 2009.

[17] L. Yu and L. Lu, "Research on test data generation based on modified genetic and simulated annealing algorithm," in Proceedings of the 2010 8th International Conference on Supply Chain Management and Information, pp. 1-3, Hong Kong, China, October 2010.

[18] G. Shang, J. Xinzi, and T. Kezong, "Hybrid algorithm combining ant colony optimization algorithm with genetic algorithm," in Proceedings of the 2007 Chinese Control Conference, pp. 701-704, Zhangiajie, China, July 2007.

[19] D. A. Linkens and H. Okola Nyongesa, "A distributed genetic algorithm for multivariable fuzzy control," IEE Colloquium on Genetic Algorithms for Control Systems Engineering, vol. 142, pp. 91-93, 1993.

[20] K. Chen, C. Han, and Z. He, "A systhesis technique for linear sparse arrays with optimization constraint of minimum element spacing," Chinese Journal of Radio Science, vol. 22, no. 1, pp. 28-32, 2007.

[21] S. Akkar, F. Harabi, and A. Gharsallah, "Linear ESPRIT-like algorithms for fast directions of arrival estimation with real structure," in Proceedings of the 2019 IEEE 19th Mediterranean Microwave Symposium (MMS), pp. 1-4, Hammamet, Tunisia, October 2019.

[22] W. Zhang, Y. Han, M. Jin, and X.-S. Li, "An improved ESPRIT-like algorithm for coherent signals DOA estimation," IEEE Communications Letters, vol. 24, no. 2, pp. 339-343, 2020.

[23] K. Almidfa, G. V. Tsoulos, and A. Nix, "Performance analysis of ESPRIT, TLS-ESPRIT and Unitary-ESPRIT algorithms for DOA estimation in a W-CDMA mobile system," in Proceedings of the First International Conference on $3 G$ Mobile Communication Technologies, pp. 200-203, London, UK, August 2000. 
[24] J. Li, Y. He, L. He, and X. Zhang, "DOD and DOA estimation for MIMO radar based on combined MUSIC and sparse Bayesian learning," in Proceedings of the 2019 International Applied Computational Electromagnetics Society SymposiumChina (ACES), pp. 1-2, Nanjing, China, August 2019.

[25] M. W. T. S. Chowdhury and M. Mastora, "Performance analysis of MUSIC algorithm for DOA estimation with varying ULA parameters," in Proceedings of the 2020 23rd International Conference on Computer and Information Technology (ICCIT), pp. 1-5, Tabuk, Saudi Arabia, May 2020.

[26] Z. Zhou-Hua, "The fourth order cumulants based modified MUSIC algorithm for DOA in colored noise," in Proceedings of the 2010 Asia-Pacific Conference on Wearable Computing Systems, pp. 345-347, Washington, DC, USA, April 2010.

[27] J. Zhuang, L. Yang, G. Ning, I. A. Hussein, and W. Wang, "Adaptive 2-D DOA estimation using subspace fitting," in Proceedings of the 2018 IEEE 23rd International Conference on Digital Signal Processing (DSP), pp. 1-5, Shanghai, China, November 2018.

[28] H. Duan, Z. Qian, and Y. Wang, "Off-grid DOA estimation based on noise subspace fitting," in Proceedings of the 2015 IEEE International Conference on Digital Signal Processing (DSP), pp. 675-678, Singapore, July 2015.

[29] Y. Fei, L. Jingqing, and Y. Zhifu, "DOA estimation methods using weighted subspace fitting technique based on immune evolutionary algorithm," in Proceedings of the 2007 8th International Conference on Electronic Measurement and Instruments, pp. 233-236, Xian, China, August 2007.

[30] J. Jin Wang, Y. Zhigang Wang, and Z. Wang, "Low complexity subspace fitting method for wideband signal location," in Proceedings of the 2008 5th IFIP International Conference on Wireless and Optical Communications Networks (WOCN '08), pp. 1-4, Surabaya, Indonesia, January 2008.

[31] Y. Guo, W. Li, J. Hu, L. Shi, X. Feng, and C. Liu, "Reducedrank covariance matrix based algorithm for DOA estimation," in Proceedings of 2014 3rd Asia-Pacific Conference on Antennas and Propagation, pp. 686-689, Harbin, China, July 2014.

[32] Y. Guo, W. Li, J. Shen, X. Xu, J. Zhang, and Y. Zuo, “A low complexity algorithm for DOA estimation based on reducedrank covariance matrix," in Proceedings of the 2014 IEEE International Conference on Signal Processing, Communications and Computing (ICSPCC), pp. 61-64, Guilin, China, August 2014.

[33] B. Liu, G. Gui, S. Matsushita, and L. Xu, "Dimension-reduced direction-of-arrival estimation based on $\$ \backslash$ ell_ $\{2,1\} \$$-norm penalty $\ell_{2,1}$-norm penalty," IEEE Access, vol. 6, pp. 44433-44444, 2018.

[34] N. W. M. Thet, M. K. Ozdemir, and A. Kachroo, "Reducedrank joint estimation of DOA with mutual coupling," in Proceedings of the 2018 26th Signal Processing and Communications Applications Conference (SIU), pp. 1-4, Izmir, Turkey, May 2018.

[35] Y. Ma, X. Cao, and X. Wang, "Multi-source off-grid DOA estimation using iterative phase offset correction in coarray domain," Digital Signal Processing, vol. 112, Article ID 102998, 2021.

[36] L. Zhang, Q. Huang, K. Liu, and Y. Fang, "Wideband DOA estimation in spherical harmonic domain using sparse Bayesian learning," in Proceedings of the 2017 36th Chinese Control Conference (CCC), pp. 5183-5187, Dalian, China, July 2017.
[37] Z. Xu, X. Wang, S. Xiao, and Z. Zhuang, "Joint spectrum estmation of polarization and space," in Proceedings of the IEEE ICNNSP', Nanjing, China, December 2003.

[38] S. Zhang, Y.-Y. Xu, and J.-S. Tang, "A robust estimation method of interferometric phase based on weighted subspace fitting," in Proceedings of the 2009 2nd International Congress on Image and Signal Processing, pp. 1-5, Tianjin, China, October 2009. 